\title{
La matriz GAGO como método de catalogación de especies invasoras
}

\author{
I. Lobato ${ }^{1, *}$ \\ (1) Ciconia Consultores Ambientales S.L. \\ *Autor de correpondencia: I. Lobato [(ivanlobato@hotmail.es]
}

> Recibido el 1 de febrero de 2011, aceptado el 23 de noviembre de 2012.

Lobato, I. (2012). La matriz GAGO como método de catalogación de especies invasoras. Ecosistemas 21(3):115-117. Doi.: 10.7818/ECOS.210.21-3.18

Cuando un problema llega a la cota de ser considerado como la segunda causa de pérdida de biodiversidad, es que ha llegado el momento de actuar, de adoptar una postura valiente y de empezar a atajar el problema desde su base, delimitando objetivos y circunscribiendo responsabilidades. Es en esta línea donde la catalogación de especies invasoras tiene mucho que decir, y por añadidura las herramientas destinadas a facilitar dicha labor. Herramientas como la matriz GAGO, la cual se presenta como una oportunidad para llevar la catalogación a escala del aquí y ahora, a través de un sistema de codificación basado en parámetros biológicos básicos y de fácil acceso documental.

Palabras clave: especies exóticas, ecología de invasiones, invasiones biológicas, catálogo

Lobato, I. (2012). The GAGO matrix as a cataloguing method of invasive species. Ecosistemas 21(3):115-117. Doi.: 10.7818/ECOS.210.21-3.18 When a problem reaches the stage of being considered as the second cause of biodiversity loss, it is time to act, to adopt a bold attitude, and to start attacking the problem at its source by setting goals and defining responsibilities. With this idea in mind, cataloguing invasive species becomes a remarkable tool to carry out this task. Tools as the GAGO matrix provide an opportunity to develop the cataloguing process for this particular time and date. The matrix is created, through a codification system based on basic biological parameters and a straightforward documentary access.

Key words: exotic species, invasion ecology, biological invasions, catalogue

\section{Introducción}

Por muy variadas que sean las causas de introducción de especies invasoras, éste es evidentemente un problema de comercio internacional (Weber 2003). En este sentido, es necesario asimilar la catalogación ambiental a los sistemas comerciales para que operen a las mismas escalas espacio-temporales y de priorización de recursos económicos y personales.

Una de las posibles debilidades que plantea la catalogación de especies invasoras es el hecho de considerar las invasiones una vez que se han producido. Ello redunda en la cadena de actuación, pues ignora la detección temprana y la rápida respuesta, para convertirse en un método de contención, que queda limitado a que las especies que ya han invadido no extiendan su ocupación. Muy estrechamente relacionado con este aspecto está la escala espacial, que por lo general suele centrarse en los procesos invasivos que ocurren a nivel nacional. Esto infravalora el dinamismo ecosistémico, ya que lo que aquí y hoy es un invasor, mañana puede ser un recurso ecológico a considerar en cualquier otro lugar. Un buen ejemplo de ello es el cangrejo rojo americano para la cuenca del Guadalquivir (Tablado 2010), del cual nadie duda que es una especie invasora. Ahora bien, si la pregunta se formula como si al ser una especie invasora ha de ser erradicada a nivel nacional, la cosa ya no está tan clara, pues eliminarlo de dicha cuenca puede generar serios desequilibrios ecológicos. De ahí la necesidad de ampliar el prisma de actuación a la escala de entorno: cuando es un invasor a erradicar, y cuando no se puede considerar su erra- dicación al menos hasta restituir las poblaciones autóctonas que ha desplazado.

También es importante destacar que, si bien el comercio incrementa las invasiones biológicas (Pimentel 2002), la lucha contra las mismas carece de un apoyo económico de semejante magnitud. Es por ello que las mejores oportunidades deben pasar por aprovechar las infraestructuras de estudio ambiental disponibles y reeducar a los efectivos existentes (Capdevila-Arguelles et al. 2006).

En consecuencia, no se trata de hacer un listado de las invasiones representativas, sino que, en clara analogía al comercio, un catálogo ambiental debe constituirse como un sistema de información codificado, de fácil acceso y que responda a las preguntas de dónde, cómo y cuándo se pueden producir las invasiones, con la mayor premura posible.

\section{Métodos}

El presente método opera con base en tres fuentes de información (matriz de competitividad o matriz GAGO, análisis de invasividad y análisis de vulnerabilidad) para cada uno de los siguientes ámbitos: fauna, flora y mixto.

La matriz de competitividad para cada ámbito de estudio es el resultado de confrontar las especies autóctonas con aquellas susceptibles de ser consideradas como invasoras, es decir, aquellas especies alóctonas detectadas en el área de estudio, en entornos cercanos o que, por su modalidad de dispersión, pueda considerarse factible su invasión. De este modo, se consigue un efecto em- 
budo en la incorporación de especies a la matriz, pasando de una lente preventiva más amplia, como son las especies susceptibles de invadir, a una óptica más precisa como es la incorporación de las especies foráneas detectadas en el propio área. Dicha confrontación atenderá a los siguientes parámetros, según trabajemos en la matriz para fauna, vegetal o mixta:

\section{Matriz de competencia para fauna:}

- Hábitats: se procederá a la comparación con base en el entorno ocupado dentro del propio ecosistema (carrizal, zona de cultivo, etc.). En aquellos casos en los que una especie en particular desempeñe sus funciones vitales en varios hábitats, serán tenidos en cuenta todos ellos, realizándose una valoración para cada uno de los mismos.

- Alimentación: se evaluará la posibilidad de competencia en cuanto a hábitos nutricionales.

- Depredación: se establecerán las relaciones entre depredadorpresa plausibles entre especies nativas y foráneas.

- Lugar de anidación-reposo: se confrontarán a las especies en función de sus preferencias por los lugares de anidación o reposo, tales como nidos o madrigueras.

- Transmisión de enfermedades: en aquellos casos en que se cuente con pruebas evidentes de que la especie exótica actúa como un vector de transmisión, los organismos implicados serán confrontados en una relación negativa para el receptor (ejemplo: el cangrejo rojo americano y sus homólogos autóctonos).

\section{Matriz de competencia para flora:}

- Zonas de vida: factores bioclimáticos sistematizados por Holdridge (1987).

- Sustrato: se estudiará la posible coincidencia con respecto a sustratos similares, bien por tipo de suelos, o bien en referencia a otros tipos de sustratos, cuando se trabaje, por ejemplo, con plantas acuáticas.

- Germinación: valoración de qué especie germina en una estación menor, de manera que si la invasora germina en estaciones iguales o inferiores a las autóctonas, se pueda hablar de factor negativo para la vegetación autóctona.

- Crecimiento: se atenderá a la velocidad del mismo, estableciéndose dos categorías (rápido y lento).

- Alelopatía/enfermedad: en aquellas especies de las que se tenga constancia de una relación alelopática (Inderjit et al. 2008) o de transmisión de enfermedades, se valorará el beneficio o perjuicio de la relación alelopática, y la negatividad de la transmisión de enfermedades.

\section{Matriz de competencia mixta:}

- Hábitats: se buscará la posibilidad de encuentro en un entorno determinado por parte de especies vegetales y animales.

- Depredación: se establecerán las relaciones depredadoras plausibles entre sendos grupos.

- Reproducción: se analizará la factibilidad de que una especie pueda interferir en la capacidad reproductora de otra, ya sea de forma directa o de forma indirecta, como, por ejemplo, depredación sobre insectos polinizadores en relación a la reproducción vegetal.

- Alelopatía/enfermedad: se considerará la evidencia de relaciones alelopáticas entre especies vegetales y animales (Hierro y Callaway 2003), así como la constatación de la transmisión de enfermedades entre ambos grupos.

En la problemática de las invasiones biológicas el tiempo es oro, y la determinación de competitividad o no, ha de hacerse de forma casi instantánea. Ante esto no queda más que recurrir a bases de datos sistematizadas, de manera que cada parámetro a evaluar se constituya en un código alfanumérico vinculando a cada especie. Por ejemplo, para fauna se podría hablar de un organismo identificado como $\mathrm{H}_{2}, \mathrm{~A}_{1}, \mathrm{~A}_{2}, \mathrm{D}_{1}, \mathrm{D}_{2}, \mathrm{~N}_{3}, \mathrm{~T}_{0}$ en relación al siguiente listado de opciones:

- $\mathrm{H}_{1}, \mathrm{H}_{2}, \mathrm{H}_{3} \ldots$ donde $\mathrm{H}_{1}=$ campos de cultivo, $\mathrm{H}_{2}=$ márgenes de ríos, $\mathrm{H}_{3}=$ subsuelo...

- $A_{1}, A_{2}, A_{3} \ldots$ donde $A_{1}=$ pequeños peces; $A_{2}=$ pequeños anfibios; $A_{3}=$ cereales...

- $D_{1}, D_{2}, D_{3} \ldots$ donde $D_{1}=$ depreda pequeños peces; $D_{2}=$ depreda anfibios; $\mathrm{H}_{3}=$ depreda pequeñas aves...

- $\mathrm{N}_{1}, \mathrm{~N}_{2}, \mathrm{~N}_{3} \ldots$ donde $\mathrm{N}_{1}=$ anida/reposa en suelos de cultivo; $\mathrm{N}_{2}=$ anida/reposa en árboles de grandes copas; $\mathrm{N}_{3}=$ anida/reposa en subsuelo blando...

- $\mathrm{T}_{0}, \mathrm{~T}_{1} \ldots$ donde $\mathrm{T}_{0}=$ no hay registrado ningún caso de transmisión de enfermedad entre las dos especies cruzadas; $T_{1}=$ se cuentan con evidencias constatadas de transmisión de enfermedades entre sendas especies.

Con respecto a la construcción de la matriz GAGO, una vez se han establecido las categorías de codificación, el cruzamiento resulta sencillo, con lo que los mismos operarios de los espacios afectados podrían elaborarla.

En referencia al proceso de evaluación, este consistirá en la asignación de tres caracteres $(\mathrm{N}, \mathrm{P}, \mathrm{y} 0)$ en relación a las especies nativas: $\mathrm{N}$ si la coincidencia de los parámetros se muestra como negativa para las especies autóctonas; $\mathrm{P}$ si la relación es beneficiosa, y; 0 si no se establece coincidencia entre parámetros.

\begin{tabular}{|c|c|c|c|c|c|c|c|c|c|c|c|c|c|c|c|c|c|c|c|c|}
\hline & \multicolumn{5}{|c|}{$\begin{array}{l}\text { Alopochen } \\
\text { aegyptiacus }\end{array}$} & \multicolumn{5}{|c|}{$\begin{array}{l}\text { Amandava } \\
\text { amandava }\end{array}$} & \multicolumn{5}{|c|}{$\begin{array}{l}\text { Ephippiorhynchus } \\
\text { senegalensis }\end{array}$} & \multicolumn{5}{|c|}{$\begin{array}{l}\text { Estrilda } \\
\text { astrild }\end{array}$} \\
\hline & H & A & D & $N / R$ & E & H & A & D & $N / R$ & $\mathbf{E}$ & H & A & D & $N / R$ & E & H & A & D & $N / R$ & E \\
\hline Actitis hypoleucos & $\mathrm{N}$ & 0 & 0 & $\mathrm{~N}$ & 0 & $\mathrm{~N}$ & $\mathrm{~N}$ & 0 & 0 & 0 & $\mathrm{~N}$ & 0 & $\mathrm{~N}$ & 0 & 0 & $\mathrm{~N}$ & $\mathrm{~N}$ & 0 & 0 & 0 \\
\hline Acrocephalus arundinaceus & N & 0 & 0 & 0 & 0 & $N$ & $N$ & 0 & 0 & 0 & $\mathrm{~N}$ & 0 & 0 & 0 & 0 & $\mathrm{~N}$ & $\mathrm{~N}$ & 0 & 0 & 0 \\
\hline Acrocephalus scirpaceus & $\mathrm{N}$ & 0 & 0 & 0 & 0 & $\mathrm{~N}$ & $\mathrm{~N}$ & 0 & 0 & 0 & $\mathrm{~N}$ & 0 & 0 & 0 & 0 & $\mathrm{~N}$ & $\mathrm{~N}$ & 0 & 0 & 0 \\
\hline
\end{tabular}

Tabla 1. Ejemplo de matriz de competencia para fauna.

Variables: Hábitat $(H)$, Alimentación (A), Depredación (D), Lugar de Anidación-Reposo (N) y Transmisión de enfermedades (E).

Valores: Positivo (P), Negativo (N) y Neutro (O).

Especies alóctonas: Alopochen aegyptiacus (Ganso de Egipto), Amandava amandava (Bengalí rojo), Ephippiorhynchus senegalensis (Jabirú de Senegal) y Estrilda astrild (pico de coral).

Especies autóctonas: Actitis hypoleucos (Andarríos chico), Acrocephalus arundinaceus (Carricero tordal) y Acrocephalus scirpaceus (Carricero común). 
Con base en esta información, se puede intuir qué especie es susceptible de ser incluida en el catálogo, a razón de que manifieste una mayoría de resultados $\mathrm{N}$ sobre las especies originarias. No obstante, será necesario acometer dos fases más para validar qué especies parten con ventaja o desventaja en este evento competitivo, pues las que interesa tratar son las relaciones de competencia que el medio natural no puede subsanar por sí solo.

Esta segunda y tercera fase de validación es lo que se denomina análisis de invasividad y análisis de vulnerabilidad, y se llevan a cabo con aquellas especies amenazadas que presenten una estrecha relación negativa con alguna especie exótica. La designación de este tipo de atributos, siempre implica cierto grado de subjetividad, razón por la cual, se recurre a una adaptación del modelo estadístico ACPF (Aguilera et al. 1996), para obtener un valor ponderado lo más fiable posible. Este método se utilizará para establecer unos patrones de mayor o menor impacto en función de los atributos destacados de cada especie.

Para el caso del análisis de invasividad, se debe procurar el acercamiento a la realidad desde el prisma del invasor, es decir, recurrir a los estudios realizados sobre la especie objeto de estudio en su región de origen o en otras zonas invadidas, para establecer cuáles de sus características originarias son potencialmente ventajosas en caso de competencia. Así se ha de valorar no solo los atributos biológicos de la especie, sino también el carácter antrópico del fenómeno, entrando en el modo de dispersión y la frecuencia con que esta se lleva a cabo.

Con base en el análisis estadístico de las invasiones precedentes, se puede asignar un valor ponderado de impacto (Valderrama 2000), es decir, cada invasión descrita es englobada en un perfil en función a las variables de reino, atributo biológico o antrópico, entorno y periodo a partir del cual un invasor comienza a producir daños. De esta manera, las cualidades o atributos que se han reconocido en la especie exótica objeto de estudio, pueden ser valorados de forma objetiva, en función del patrón global seguido por las invasiones genéricas.

De forma paralela, se lleva a cabo el análisis de vulnerabilidad, que trata de describir los puntos débiles del ecosistema. En dicho análisis se recurre a la información previa sobre especies amenazadas en el espacio natural de estudio, y se evalúan las características de dichas especies que han derivado en su situación actual, (pérdida de hábitat, especialización nutricional, etc.) de manera que, en caso de establecerse coincidencia entre un competidor por alguno de estos factores, se considere tal coincidencia como un gravamen que pese sobre la balanza competitiva, señalando como perdedor a la especie amenazada.

Al igual que en el caso anterior, tales atributos son valorados en función del análisis estadístico de las especies amenazadas, las cuales en este caso son englobadas en sus respectivos perfiles en relación a las variables de reino, cualidad, entorno y periodo en el que comienzan a verse afectada por el daño.

Una vez que se han asignado los valores a las distintas cualidades, se seleccionan aquellas cualidades que presenten un mayor valor de impacto, y son cruzadas en función de la relación entre especie amenazada y exótica. De manera que, si del cruzamiento de los valores de ambas categorías, se obtiene un valor superior al establecido como límite, nos encontramos ante una especie exótica a catalogar como invasora.

Pese a los intentos por reducir la subjetividad en este tipo de decisiones, la elección de la información empleada va a mediar en gran medida en los resultados obtenidos. Ante esta situación, solo cabe estandarizar la búsqueda de información, priorizando las fuentes oficiales o con respaldo académico, las informaciones más restrictivas y más concretamente para el caso de las especies exóticas, los datos recogidos sobre entornos lo más similares posible al área de estudio propia.

Así pues, tras el tratamiento siempre de información previamente elaborada para otros fines, se puede determinar la inclusión de un organismo en el catálogo a tenor de su predisposición a competir en un evento ecológico y su relación vulnerabilidad-invasividad.

No obstante, la catalogación es solo un paso previo, pues tan importante como catalogar, es estar en disposición de decidir si es necesaria una actuación sobre el medio o no. Esto puede resolverse con base en la resolución de las siguientes formulas:

$$
D \times R=C ; S \times A=H ; C+H=X ; I \times V=P R ; X \times P R=M
$$

\section{Donde:}

$D=$ Daño, se le asigna un valor que oscila entre -5 para aquellos casos en que la matriz GAGO arroje varios casos de relaciones positivas con especies amenazadas, y un valor de 5 para aquellos casos donde la especie invasora incida de forma negativa sobre varias especies amenazadas.

$\mathrm{R}=$ Reemplazo, contempla valores que van de 1 a 5 en función de la dificultad de reemplazar a las especies afectadas por la invasión, resultando el valor 5 la máxima dificultad.

$\mathrm{S}=$ Sector dañado, depende de que los efectos adversos recaigan sobre el sector social, económico, ecológico o sobre varios de ellos simultáneamente, recibiendo un valor entre 1 y 5 , siendo este mayor cuanto mayor es el número de sectores afectados.

$A=$ Área, representa la extensión susceptible de verse afectada, valorándose con valores entre 1 y 5 según el tamaño de la misma.

I= Invasividad, se corresponde con el análisis de invasividad, otorgándole un valor entre 1 y 5 en relación al máximo valor ponderado obtenido en dicho análisis.

$V=$ Vulnerabilidad, procedente del análisis de vulnerabilidad, equipara el máximo valor ponderado a una escala de 1 a 5 .

$\mathrm{M}=$ necesidad de adoptar medidas.

En consecuencia, si tras los cálculos propuestos $M$ supera un valor prefijado, nos encontramos ante una especie invasora que requiere de medidas inmediatas. Por el contrario, si este valor es inferior al mismo, pese a tratarse de una especie invasora, podemos postergar en el tiempo la actuación. De esta manera se consigue priorizar los recursos en función de las necesidades de actuación.

\section{Referencias}

Aguilera, A.M., Ocaña, F.A., Valderrama, M.J. 1996. Análisis en componentes principales de un proceso estocástico con funciones muestrales escalonadas. Questiió: Quaderns d'Estadística, Sistemes, Informatica i Investigació Operativa 20 (1):7-28.

Capdevila-Arguelles, L., García, A.I., Orueta, J.F., Zilletti, B. 2006. Especies exóticas invasoras: diagnóstico y bases para la prevención y el manejo. ICONA, Organismo Autónomo de Parques Nacionales, Ministerio de Medio Ambiente, Madrid. España. ISBN 9788480146678

Hierro, J.L., Callaway, R.M. 2003. Allelopathy and exotic plant invasion. Plant and Soil 256:29-39.

Holdridge, L.R. 1987. Ecología basada en zonas de vida. Humberto Jiménez Saa (Trad.). Instituto Interamericano de Ciencias Agricolas, San José, Costa Rica. ISBN 9789290391319

Inderjit, T.R., Seastedt, R.M., Callaway, J.L., Pollock; Jasleen Kaur. 2008. Allelopathy and plant invasions: traditional, congeneric and bio-geographical approaches. Biological Invasions 10:875- 890.

Lobato, I. 2011. Invasiones biológicas: Diagnosis y solución. Gerüst Creaciones, S.L., Torrejón de la Calzada. Madrid, España.

Pimentel, D. 2002. Biological Invasions. Economics and Environmental Costs of Alien Plant, Animal, and Microbe Species. CRC Press, Boca Raton, FL, USA.

Valderrama, M.J., Aguilera, A.M., Ocaña, F.A. 2000. Predicción dinámica mediante análisis de datos funcionales. La Muralla-Hespérides. Madrid, España.

Weber, E. 2003. Invasive Plant Species of the World. A Reference Guide to Environmental Weeds. CABI Publishing. Wallingford, Oxfordshire, UK.

Tablado, Z., Tella, J.L. Sanchez-Zapata, J.A., Hiraldo, F. 2010. The paradox of the long-term positive effects of a north american crayfish on a European community of predators. Conservation Biology. DOI: 10.1111/j.1523-1739.2010.01483.x 